\title{
Risk-based reboot for global lab biosafety standards
}

first publication of LBM coincided with the year when polymerase chain reaction (PCR) was invented. Since then, over many decades and three editions of LBM, many common biosafety concepts and practices have been applied without assuming the availability of technologies such as molecular testing that, since it does not require propagation of the pathogen, poses considerably less likelihood for exposure compared to conventional culture-based diagnostics. ganisms in labs. But as technologies continue to evolve, and with them potential threats and benefits to lab safety, so too must approaches to biosafety. With revision toward the $4^{\text {th }}$ edition of LBM underway, we propose a shift in focus to a risk-based, technology-neutral and cost-effective approach to biosafety, making sure that lab facilities, safety equipment, and work practices are locally relevant, proportionate, and sustainable. This will allow more flexibility in lab design, reduce focus on pathogen risk groups and biosafety levels as the de facto starting point of lab considerations and place more emphasis on human factors and worker training. Improved sustainability of lab operations through lower construction and operating costs, particularly in resource-limited settings, may pave the way for equitable access to clinical and public health lab tests and to biomedical research opportunities, without compromising safety.

The LBM has been in broad use at all levels of labs and biomedical sectors globally, serving as de facto standards that represent best practices, especially where resources are less plentiful and regulatory frameworks are less well developed. The

\footnotetext{
${ }^{1}$ World Health Organization, Geneva, Switzerland. ${ }^{2}$ Health \& Safety Laboratory, Buxton, UK. ${ }^{3}$ Institute of Virology and Immunology, Mittelhäusern, Switzerland. ${ }^{4}$ Public Health England, Salisbury, UK. ${ }^{5}$ Public Health Agency of Canada, Ottawa, Canada. ${ }^{6}$ Mahidol Oxford Tropical Medicine Research Unit, Bangkok, Thailand. ${ }^{7}$ Centre for Tropical Medicine and Global Health, Nuffield Department of Clinical Medicine, University of Oxford, Churchill Hospital, Oxford, UK . ${ }^{8}$ Centers for Disease Control and Prevention, Washington, DC, USA. ${ }^{9}$ National Institutes of Health, Bethesda, MD, USA *Correspondence to: kojimak@who.int
}

\section{PRECAUTIONARY AND PRESCRIPTIVE}

Under earlier editions of the LBM, local risk assessment was not regularly and extensively practiced, which necessitated a more precautionary and prescriptive approach to defining safety criteria for pathogens and laboratory facilities. Biological agents that have minimal or no available preventative or therapeutics, such as Ebola virus, have typically been handled only in high containment facilities, irrespective of the procedure, which might have contributed to unnecessary proliferation of costly high containment labs.

Local risk assessment was encouraged in the third edition of the LBM in 2004, but at the same time it did not adequately address what had become overly simplified implementation of biosafety programs born of the tendency to equate pathogen Risk Groups (RGs, or Hazard Groups) with laboratory Biosafety Levels (BSLs, or Containment Levels). RGs and BSLs, which both have the same numerical nomenclature, have incorrectly been assumed to be essentially equated, e.g., all work with RG2 pathogens must be conducted at BSL2; RG3 at BSL3; RG4 at BSL4.

The consequence of this equation is that pathogen and local risk assessments are not routinely carried out, missing opportunities to optimize the means of risk control proportionate to the assessed risks. While RG may be a useful tool for national regulatory regimes, applying them universally does not take into account national epidemiological profile, such as endemicity, nor does it necessarily consider local application of procedures and techniques.
When the concept of RGs was introduced in the LBM for national oversight regimes, there was clear guidance that RG determination was to be specific to a particular jurisdiction, based on local/national conditions. But this approach was lost over time by many applying this concept internationally. A major source of confusion is inconsistency in the description of pathogen RGs and BSLs between countries (1).

\section{ENGINEERED AND UNSUSTAINABLE}

At higher BSLs there has been an emphasis on strict application of detailed engineering controls, but much less consideration has typically been given to procedural and human factors, despite the fact that the same pathogen could pose considerably different risks according to the procedures to be undertaken. Consequently, structural systems such as complicated and costly centralized ventilation units, complex waste disposal, and building automation systems, tend to take precedence over risk assessment, workforce development, and training on good microbiological practices and procedures (GMPP).

Yet a review of recent laboratory acquired infections (LAIs)(2) demonstrated that most were caused not by malfunctions of engineering controls, but by human factors such as improper use of personal protective equipment (PPE), disregard for or inadequate risk assessments, and lack of standard operating procedures (SOPs) and/or properly trained staff. The best designed and most engineered laboratory is only as good as its least competent worker.

For routine diagnostics, and pathogen research not involving high concentrations and large volumes of infectious materials, there is no evidence that using complex biological containment facilities will provide a safer working environment, while reliance on such facilities will most certainly increase costs. Facilities of complex design are costly to build, operate, and maintain, particularly those with centralized ventilation systems or complex waste disposal systems. Day-today running and maintenance costs of high containment laboratories are often not pri- 
oritized during budgeting and planning.

Most laboratories, especially those in resource-limited countries, cannot reasonably afford to build, run, and maintain such facilities without long-term donor or external support, but often strive to acquire them based on past global trends to building containment labs, where local risk assessment was not a routine practice. Where there is a lack of financial resources for laboratory services, running complex containment facilities can take valuable and limited resources away from day-to-day laboratory operations, potentially leaving lower-profile laboratories more vulnerable to adverse occurrences. The lack of technical resources, such as appropriately trained and locally available engineers, further complicates these challenges.

\section{HUMAN-FOCUSED AND FLEXIBLE}

In the new LBM, all risk mitigation measures should be selected using a risk- and evidence-based, rather than BSL check-list, approach. They should be informed by the consequence and likelihood of exposure to a pathogen. The same principles apply when considering emerging technologies in the context of dual use research of concern, and synthetic biology; biosecurity considerations should be proportionate to the assessed risks to complement biosafety effectively, sharing the same goal to prevent unnecessary release and exposure of biological materials, be it accidental or deliberate $(3,4)$. A risk-based approach also underpins the revised World Organisation for Animal Health (OIE) standard for managing biological risk in veterinary labs and animal facilities (5).

This multi-factorial assessment includes criteria such as: route(s) of infection; pathogenicity and infectious dose; prophylaxis or vaccine availability; disease severity and mortality; contagiousness; endemicity; high risk laboratory procedures (i.e., work with aerosols, high titres or volumes, sharps, animals); laboratory workforce competency; highly susceptible individuals/hosts; volume and titre of the biological agent(s) being produced/handled; biosecurity (potential for misuse/weaponization).

In lieu of defined biosafety levels, the revised LBM has proposed "core requirements" are proposed, a combination of common biosafety elements to be implemented and used, and a minimum requirement for safe work during the majority of laboratory procedures. These include the laboratory facility and equipment, but also procedures and appropriately trained and competent staff, which too often received disproportionately less attention.

Where the local risk assessment necessitates, heightened control measures such as the use of biological safety cabinets (BSCs), extra PPE, and in rare cases high containment would be used to mitigate the identified risk(s) of exposure to the biological agent(s) being handled. This reinvented definition is intended to be more continuous than the discrete classification of BSL. The intent is to choose the right set of safety measures to control the identified risk(s), and not rely on a predefined "universal" set that may be an overdesign based on the pathogen and/or activities proposed.

\section{GLOBALIZING BIOSAFETY}

The need to update international lab biosafety guidance is part of a broader initiative to globalize biosafety, emphasizing principles and approaches that are accessible to countries spanning a broad range of financial, technical and regulatory resources. The WHO revised the International Health Regulations (IHR) in 2005 "to help the international community prevent and respond to acute public health risks that have the potential to cross borders and threaten people worldwide". This requires all 196 States Parties to be well prepared for potential outbreaks and new diseases, including prompt early diagnosis to enable infection prevention and control that should entail safe and secure handling and transport of infectious agents.

Biosafety and biosecurity are recognized as one of the 19 technical areas in the IHR Joint External Evaluation Tool (6).

Countries that have or are developing national oversight regimes for biosafety and biosecurity should approach oversight with a goal to be as flexible as possible to allow for multiple solutions and mitigation measures. If risk groups have been developed at the national level and embedded within legal frameworks, there should be an opportunity for periodic updates so that new evidence that supports changes to the risk profile of a pathogen are easily incorporated into a risk group change. Oversight systems should consider the procedures being conducted in facilities, and how they contribute to or reduce risk, and be addressed accordingly.

Whether prescribed or recommended, specific mitigation measures may best be applied through mechanisms with greater flexibility than legislation, such as guidelines or standards. Oversight bodies should actively engage with biosafety practitioners within their jurisdictions, to ensure consideration of practical input from the field, to maintain local and national relevance.

The process of revising the LBM and preparing accompanying technical guidance is ongoing. A critical element is an extensive consultation process of a broad range of stakeholders. It is our hope that our proposal here can inspire some readers to contribute constructively to this process.

\section{REFERENCES AND NOTES: \\ 1. D. Tian, T. Zheng, PLoS One. 9, e101163 (2014). \\ 2. N. Wurtzet al., EurJ Clin Microbiol Infect Dis. 35, 1247 1258 (2016). \\ 3. World Health Organization (WHO), Biorisk management - Laboratory biosecurity guidance(WHO, Geneva, Swit- zerland, 2006) \\ 4. European Committee for Standardization (CEN), CEN Workshop Agreement CWA 15793 Laboratory biorisk management (CEN, Brussels, Belgium, 2011). Expired but being transformed to an International Organization for Standardization (ISO) international standard. \\ 5. World Organisation for Animal Health (OIE), "Biosafety and biosecurity: standard for managing biological risk in the veterinary laboratory and animal facilities" in Manual of Diagnostic Tests and Vaccines for Terrestrial Animals (OIE, Paris, France, 2015), chap. 1.1.4. \\ 6. World Health Organization (WHO), Joint external evalu- ation tool: International Health Regulations (2005) (WHO, Geneva, Switzerland, 2016)}

\begin{abstract}
Acknowledgments:
The authors thank relevant WHO staff for their support of this revision project, in particular S. Cognat, F. Fuchs and G. Rodier. We are grateful for financial assistance from the Biosecurity Engagement Program of the United States Department of State and the Global Partnership Program of Global Affairs Canada. S.D.B. is supported by the Wellcome Trust of the United Kingdom. The authors alone are responsible for the views expressed in this article and they do not necessarily represent the views, decisions or policies of the institutions with which they are affiliated.
\end{abstract}

10.1126/science.aar2231 